\title{
Images crossing borders: image and workflow sharing on multiple levels
}

\author{
Peeter Ross • Hanna Pohjonen
}

Received: 4 August 2010 /Revised: 21 October 2010 /Accepted: 13 December 2010/Published online: 12 January 2011

(C) European Society of Radiology 2011

\begin{abstract}
Digitalisation of medical data makes it possible to share images and workflows between related parties. In addition to linear data flow where healthcare professionals or patients are the information carriers, a new type of matrix of many-to-many connections is emerging. Implementation of shared workflow brings challenges of interoperability and legal clarity. Sharing images or workflows can be implemented on different levels with different challenges: inside the organisation, between organisations, across country borders, or between healthcare institutions and citizens. Interoperability issues vary according to the level of sharing and are either technical or semantic, including language. Legal uncertainty increases when crossing national borders. Teleradiology is regulated by multiple European Union (EU) directives and legal documents, which makes interpretation of the legal system complex. To achieve wider use of eHealth and teleradiology several strategic documents were published recently by the EU. Despite EU activities, responsibility for organising, providing and funding healthcare systems remains with the Member States. Therefore, the implementation of new solutions requires strong co-operation between radiologists, societies of radiology, healthcare administrators, politicians and relevant EU authorities. The aim of this article is to
\end{abstract}

P. Ross $(\bowtie)$

Institute of Clinical Medicine, Tallinn University of Technology,

East Tallinn Central Hospital,

Pärnu mnt 104,

11312 Tallinn, Estonia

e-mail: Peeter.Ross@itk.ee

H. Pohjonen

Institute of Clinical Medicine, Tallinn University of Technology,

Akadeemia tee 15,

12618 Tallinn, Estonia

e-mail: Hanna.Pohjonen@rosalieco.fi describe different dimensions of image and workflow sharing and to analyse legal acts concerning teleradiology in the EU.

Keywords Teleradiology $\cdot$ Radiology information systems (RIS) · Workflow · Legislation as topic · Information dissemination

\section{Introduction}

The sharing of medical images, relevant clinical data and reports between healthcare organisations or between healthcare organisations and the citizen is changing dramatically: in addition to linear point-to-point connections, the matrix type of many-to-many connections is emerging. The latter allows patient information to be shared across workspaces and communities, as well as medical experts to be accessed across state borders. This new type of communication, endorsed by eHealth tools, has been noticed by the European Union (EU) and national authorities to be an instrument to be used to shorten waiting lists, to optimise the use of resources and to enable productivity gains [1-3].

National electronic patient data exchange platforms are being built all over Europe to support data sharing across organisations in a trusted and secure way. There is also the European eHealth Project, called epSOS, to connect national platforms to each other in order to view patients' summaries and e-Prescription/e-Dispensing data across national boundaries. The epSOS project aims to demonstrate that it is feasible for any EU Member State providing eHealth services for its residents to also offer these services when they travel abroad [4].

Teleradiology has been used for remote consultations for approximately three decades $[5,6]$. Despite the long 
tradition and the fact that it is currently the most advanced telemedicine service, the number of healthcare institutions using teleradiology has been limited in Europe. Only in recent years have the development of information technology (IT) and well-established standards in diagnostic imaging enabled the use of teleradiology to a larger extent. Image sharing leads to the next service level, which is imaging-related workflow sharing. However, there is still a considerable amount of hesitancy in implementing teleradiology in daily practice throughout the radiology and healthcare community. The main barriers to the implementation of teleradiology service crossing organisational or state borders are technical and semantic interoperability and legal issues.

Although the implementation of the new type of workflow is strongly supported by the EU authorities, the responsibility for making eHealth a success remains mainly with the Member States. To achieve the full potential of telemedicine the Member State has to be involved actively in integrating it into health services [1].

In November 2008 the European Commission published a communication on telemedicine for the benefit of patients, healthcare systems and society. The main aims of this communication were to establish legal clarity in eHealth and telemedicine, to solve interoperability and technical issues, to facilitate market development, and to build acceptance of telemedicine services [1].

The purpose of this article is to describe the different dimensions of image and workflow sharing that have been allowed by the recent development of an electronic healthcare environment. There is also a review of the legal acts concerning teleradiology in the EU.

\section{Dimensions and evolution in image and workflow sharing}

In linear workflow, the image information is carried either by the healthcare professional or the patient. The information is available only in one place at one time. This illustrates the situation in diagnostic imaging before the introduction of digital images - the image was taken in the radiology department and after that shared with only a few people during a long time span.

Today, medical images are available from any location in the world straight after acquisition for the patient and for healthcare professionals, if they have the relevant entitlement. This is enabled by secure communication provided by picture archiving and communication systems (PACS), digital archives and secure data exchange platforms. This allows image sharing inside the organisation, between the organisations in one region or across country borders, and also gives the patient the possibility to have instant access to the images. Consequently, image sharing leads to shared workflows between the different parties.

There are new emerging standards and profiles to support sharing of medical information. Integrating the Healthcare Enterprise (IHE) standards for document and image sharing are growing fast. The Cross-Enterprise Document Sharing (XDS) registers and shares electronic health-record documents between healthcare enterprises. Cross-Enterprise Document Sharing for Imaging (XDS-I) extends XDS to sharing images, diagnostic reports and related information across a group of care sites [7].

In the following, the different levels of image and workflow sharing are described.

\section{Sharing images inside the healthcare organisation}

Although the production of images remains mainly the responsibility of the radiology department, as in previous times, the digital images are available to the referring physicians all over the healthcare institution right after acquisition. This is decreasing the management time of stationary and ambulatory care patients. Quick image sharing places higher demands for image quality and the image management process but does not require any additional legal measures as long as images do not cross the organisational borders. However, administration of user rights and management of log files are new tasks for the organisation and need complementary resources. Sharing of images across the organisation is almost a must everywhere that PACS are installed. The radiology departments or groups use shared workflow inside the institution to re-engineer the imaging and reporting processes according to the imaging technique, anatomical or clinical subspecialty, emergency, or other agreed workflow feature.

\section{Sharing images between healthcare organisations}

Sharing images between the healthcare organisations creates new challenges regarding quality control, trust, workflow management, legal issues, reimbursement and interoperability of electronic patient records (EPR), radiology information systems (RIS) and PACS. The most important challenge is to ensure that reporting of images outside the organisation does not in any way reduce the quality of radiology services provided to the citizen [6]. The benefits of inter-organisational image sharing are: the availability of sub-specialist opinions and specialist case transfer in case there is a lack of particular local knowledge, provision of on-call emergency reporting and radiological services to remote rural communities, improvement of the reporting capacity of healthcare 
organisations, and balancing the workload across radiology departments $[6,8]$. The technical issues in sending and receiving the images seem to be almost solved: well established digital imaging and communication standards and standard profiles like XDS-I allow image sharing between organisations [7].

Modern IT systems allow the building of a global shared worklist across different PACS and RIS, not only for the viewing and sharing of data but also for truely shared workflow. This opens new options for radiology providers. Sharing of the workflow can be achieved without replacing the existing local or regional investments but implementing standardised software and platforms for sharing. With the global worklist it is possible to avoid the manual management of who reads what and where [3,9]. The study is locked from the global worklist when being read but unlocked and updated after reading is complete. Radiologists serving multiple healthcare facilities can accomplish remote reading and reporting across large geographical areas. This is an excellent way to balance workload locally or regionally between sites with different RIS or PACS [3].

Cross-enterprise document sharing can also provide reporting radiologists with access to other relevant clinical data than available in RIS. Sharing EPR case summaries could be a valuable asset in the case of complicated clinical situations. This is especially valid when the patient has a history of visiting multiple healthcare institutions.

A good example of countrywide usage of shared workflow is Ireland, where they are deploying a nation-wide system to enable any examination to be viewed and reported anywhere in the country regardless of the original place of imaging. Another good example is the project in northern Finland, where a collaboration platform can be used to carry out reporting between multiple hospitals [10]. Through the reporting platform there is a possibility to carry out reporting and consultations over the Internet using a virtual private network channel, to provide a forum for specialists in the region to view and report images, irrespective of their location. In the Western Health Region of Norway, an integration platform is used to integrate four RIS and five PACS within 15 hospitals and several private enterprises. The solution is based on sending and receiving request and report messages in HL7 or CEN/XML format. Through desktop integration with the local RIS and PACS, the integration platform offers secure, simple and fast interorganisational access to images and relevant clinical information. The solution provides safe Web access to radiological requests, reports and images, combined with an option of retrieving this information into local systems when needed [11].

The reimbursement of teleradiology services in interorganisational workflow sharing is an issue that often makes difficulties and is seldom solved automatically with the clinical set-up. Usually the financial software of the healthcare institution is not integrated with the clinical software, thus making the financial management of the imaging between the organisations difficult. Until recently, reimbursement has been agreed by bilateral contracts between two healthcare organisations. However, new models of brokering reimbursement schemes are evolving. There are examples of inter-organisational brokering services, so-called eMarketplaces, under development in the EU and North America. The partially EU-funded market validation project, R-Bay, concerning cross-border teleradiology was one of the first dealing with implementation of an eMarketplace [12].

The eMarketplace has a brokering function and gets paid according to transactions through the eMarketplace. It is a controlled way to practise teleradiology with transparent quality assurance, centralised certification databases and uniform data privacy and security policies. In this brokering service set-up, end-customers and teleradiology providers from different organisations can interact through a central connection platform. The concept follows the many-tomany connection principle. With such an integrated platform, the whole market opens up with one integration only and the stakeholders can compare prices, availabilities, response times, quality, sub-specialities, etc. Today the same concept is deployed in Denmark and England, where one of the eMarketplace research projects is now turning more commercial.

In North America, similar shared workflow implementations are in commercial use. Companies like Telerays or Virtual Radiologic in the United States (USA) and Real Time Radiology in Canada integrate hospitals, imaging centres and radiologists to work together [13-15]. A single integrated worklist, viewer and reporting system are provided. Telerays built their concept on an online auction and a reading room in which radiologists can download radiology cases and upload final interpretation reports for placement in the patient's medical record. Only radiologists pre-approved by the hospitals and imaging centres are allowed to bid on the contracts. Each radiologist must answer questions about their educational background and practice history as well as standard disclosure questions including previous licensure issues, sanctions, etc.

Transfer of image-related data outside the imaging facility requires additional identification, safety and security measures. Still, in most cases, at least in Europe, this is usually solved by bilateral contracts between the organisations addressing licensing, liability, and accreditation and registration of imaging services and professionals. Unfortunately the complex legal environment, the need for multiple contracts in the case of point-to-point connections and resource demanding integration of EPR, RIS and PACS has made an inter-organisational shared workflow a 
demanding task - even despite the availability of internationally agreed IHE standards like XDS or XDS-I. There is a clear need for new models of management of cross-site imaging workflow, including the eMarketplace.

\section{Sharing images across country borders}

The basic components that need to be addressed in an interorganisational setting (quality and trust, interoperability, identification, security, legal issues) apply also to the crossborder settings. However, the legal issues are more complex because of the differences in healthcare and particularly telemedicine regulations among the Member States. Additional issues to be addressed are semantic interoperability and language.

The incentives of the healthcare provider to use a cross-border imaging service are to gain access to subspecialists, to solve the shortage of radiologists or to lower the reporting costs. In addition to the need for the new models of management of imaging workflow described in the inter-organisational setting the international cross-border setting needs a reliable solution for translating reports, which is currently not commercially available in teleradiology [6].

In North America, shared workflow implementations across national borders are in commercial use. Nighthawk Radiology Services uses image reading centres in Australia and Switzerland to decrease night time reporting in emergency radiology, utilising the time difference [16]. Final reports are dictated by the local group the next morning, when previously obtained imaging studies and additional medical histories are available [17].

\section{Sharing images with citizens}

Digital medical images have been distributed to the patients in the linear way of sharing-images have been either printed on film or burned onto CD or DVD. This makes the citizen an information carrier and does not allow sharing of images using the matrix set-up. Only recently the advances in user identification, data privacy and security, and streaming technology have allowed the citizen to access personal image files in PACS. In this model the citizen is no longer the carrier of image information but can open images irrespective of the location or time. Sharing digital images via the Web with patients is a new feature in medical imaging management. Secure user identification by PACS, RIS or EPR is the key element. Access to the images opens up new options for the patient: sharing images with other clinical specialists and improving his or her knowledge of the treatment process.
In Estonia, there is a nationwide solution for the archiving and sharing of medical data called the Estonian Health Information System (EHIS). Radiology reports and image links are also stored in the EHIS. Authentication of the person accessing the EHIS is based on the ID card, which is a compulsory document for the purposes of personal identification, including electronic identification, in Estonia. Although the images are not yet accessed through the EHIS, some of the hospitals allow patients to access their images in PACS using ID card identification. Besides secure identification, a user-friendly viewing application is provided to the patient so that he or she can view the images from their own computer without requiring a wide bandwidth or advanced personal computer properties. The viewing solution for the patient uses adaptive streaming technology. Acceptance of this feature by citizens has been surprisingly high. In 2009 the average number of patient queries was 800 per month for the radiology department, making approximately 170,000 images annually.

A similar concept has been implemented in the Center for Diagnostic Imaging (CDI), USA. The patient provides the CDI with their email address, which triggers the ability to set up a password-protected account. Through that account,he or she can pre-register on-line to receive preparation and appointment instructions, to book the time for imaging procedures, to submit personal medical data and also view their diagnostic reports and images. CDI consists of more than 50 centres in ten States nationwide (Steve Fisher, Center For Diagnostic Imaging, St. Louis Park, Minn., USA, personal communication).

\section{Legal aspects of telemedicine at the $\mathbf{E U}$ level}

EU legislation regulating teleradiology and eHealth services consists of multiple directives and legal documents, which makes the interpretation of the legal system in the EU extremely complex. Besides EU directives, there are additional legislative documents like the EU Communication on Telemedicine [1] and the White Paper in Teleradiology issued by the European Society of Radiology (ESR) [18]; however, these have no legal power.

The definitive view of ESR regarding teleradiology is that a radiological act must always be defined as a medical act, even if it is performed remotely using information and communication technology tools [19]. Otherwise teleradiology could be practised by non-radiologists or even by non-physicians [20]. In some EU countries, teleradiology is not considered a medical act. In Germany, teleradiology faces legal restrictions. In the case of primary reading, special governmental permission is needed, which is valid for a maximum of 3 years. In general, permission should be 
restricted to night-time and weekends; only in special cases of insufficient local resources could teleradiology also be allowed in the daytime. At the location of the imaging a specially educated physician has to be present to approve the indication and to control the procedure. The teleradiologist must be a radiologist or in the case of X-ray examinations a specially trained and licensed physician (Prof. Dr. Thomas Hackländer, Solingen, Germany, personal communication) [21].

From the EU legislation point of view, telemedicine is both a health service and a social information service. Telemedicine falls under secondary EU legislation, in particular the $E U$ Directive on Electronic Commerce [1, 22].

The European Court of Justice has stated that health services are considered to follow the fundamental principle of freedom of movement. This applies despite the subsidiarity principle of healthcare provision or the way in which it is organised or financed [1,23]. This includes the freedom for citizens to seek and receive medical treatment from another Member State, regardless of how the service is delivered, i.e. also by telemedicine.

Regarding the harmonisation of technical interoperability the EU Directive on Technical Standards and Regulations establishes a procedure that imposes an obligation on Member States to notify the Commission and each other of all draft technical regulations concerning products and information society services, including telemedicine, before they are adopted by national laws $[1,24]$.

The directive on electronic commerce defines rules for the provision of telemedicine services both within and between Member States. For teleradiology services, the service offered by a professional must comply with the rules of the Member State of origin. This applies to teleradiology services between the healthcare providers, radiology groups and/or private radiologists and follows the country of origin principle.

Telemedicine is also recognised in the proposal for a Directive on the Application of Patients' Rights in CrossBorder Healthcare [1, 21, 25]. This directive is currently under review. The proposal addresses patients' cross-border mobility including their ability to access services across borders. If adopted, the directive would require the Commission and Member States accordingly to take measures to ensure the interoperability of means for the provision of eHealth services, including teleradiology. It is stated that when healthcare is provided in a Member State other than that where the patient is an insured person, such healthcare is provided in accordance with the legislation of the Member State where treatment takes place. The abovementioned healthcare is provided in accordance with standards and guidelines on quality defined by the Member State where treatment takes place [25]. Also, ESR demands that teleradiology should be the responsibility of the
Member State where the patient undergoes the imaging procedure or telemedical referral [19].

EU Directives on the Processing of Personal Data and the Protection of Privacy in the Electronic Communication Sector [26, 27] specify a number of specific requirements relating to confidentiality and security that telemedicine and all other interactive on-line services have to meet in order to safeguard individuals' rights. These acts also provide requirements for providers of electronic communication services over public communication networks to ensure confidentiality of communications and security of their networks [1].

Cross-border teleradiology raises the question of the recognition of professionals between the Member States. ESR underlines that even if the recognition of professionals is legally regulated by the Directive on the Mutual Recognition of Qualifications it is important to establish standardised European training curricula and structures for all radiologists $[19,28]$. The recognition of professional qualifications by the host Member State allows radiologists to practice radiology, including teleradiology, in that Member State under the same conditions as its nationals [1]. The directive establishes the criteria for a set of regulated professions according to which qualifications obtained in one Member State are recognised by another but does not indicate adequate monitoring and accreditation mechanisms. ESR supports the 2007 Portugal Agreement which deals with exchanging registers of medical professionals of Member States and sharing on-line information about disciplinary and criminal findings against health professionals [29].

The legal relations between the patient and healthcare provider are governed by national and EU legislation. Considering diagnostic services or treatment, the patient has almost always an insurance relationship with a local healthcare provider in the Member States. Consequently, issues of medical errors or other conflicts are elaborated according to the Member State's jurisdiction. This does not change when teleradiology is practised within the EU. In the case of a conflict the patient can sue the local healthcare provider, and if the local provider sees that the medical error is caused by a teleradiology provider, it can sue the teleradiology provider [20]. The responsibilities of both parties are usually fixed in the teleradiology service contract. If the teleradiology provider resides outside the $\mathrm{EU}$, the above-mentioned principles do not apply unless contractually fixed.

Regarding the patient's informed consent for teleradiology, it is not regulated by the EU legislation. Some countries, like Finland, demand the patient's informed consent when patient data cross the organisational borders. The Proposal of Cross-Border Healthcare Directive states that patients should be fully informed about the various 
options regarding diagnosis and treatment in order to make a conscious choice between the various options [20].

More legal clarity is needed in the next few years in the area of teleradiology and eHealth. This will be obligatory to achieve the anticipated benefits of new data-sharing models in healthcare.

\section{Discussion}

The benefit of digitalisation in healthcare is the possibility to share medical data, including medical images, and workflows between healthcare providers or healthcare providers and patients irrespective of the place or time. Sharing of images or workflows can be done on different levels-inside the organisation, between organisations inside the country or across the country borders, and with citizens.

The EU is driving the change in the organisation and management of healthcare. Implementation of eHealth and telemedicine to enable change is gradually gaining support at the national, regional and local levels. The drivers for implementation of new services are:

- Increasing healthcare quality

- Increasing availability (easier and faster access to care)

- Decreasing the costs of healthcare services

The role of teleradiology in current radiology settings has been discussed thoroughly at international meetings and in scientific papers in recent years. The extent of how much teleradiology and remote reporting will be used in the future may vary by region or country but it is widely accepted that teleradiology will remain an option in the provision of radiology services [8, 17, 30].

The challenges that arise when images cross borders depend on the level of data sharing. Mostly they deal with interoperability, legal clarity, quality and trust. Depending on the level of sharing the interoperability issues are technical, organisational (including the seamless medical data exchange between different information systems), or semantic (including language). In inter-organisational teleradiology the workflow management and interoperability of EPR, RIS and PACS is an issue. Barriers without crossing state borders tend to be mainly technical. At the crosscountry level the legal and language issues dominate.

However, to re-engineer the workflow in a manner that patients' medical data and also the care workflow are shared between the different organisations to achieve the best care for a patient is still a challenge. For instance, the eMarketplace type of many-to-many connections in teleradiology is emerging slowly. One of the reasons for this is the inability of IT systems to offer sufficient integration in many cases. Previous projects to implement eMarketplace- type applications have not been very successful with regard to technical interoperability [6]. The problem will be adequately solved only if all parties are using XDS and XDS-I standards. Unfortunately the problem remains unsolved until the older systems without XDS-I support are replaced. The situation is somehow similar to the time before the introduction of the DICOM standard. The other reason for the slow emergence of eMarketplaces seems to be the reluctance of decision makers at the regional or healthcare institution level to accept the change in the information sharing paradigm. The mind-set is changing more slowly than changes in technology.

The future solution to implementing teleradiology services at the inter-organisational and international levels could be the eMarketplace, however. It enables teleradiology to be practised in a controlled way, allowing transparent quality assurance and uniform data privacy and security policy. Through centralised quality assurance, for example, every tenth examination can be automatically double-read and any discrepancies discussed. The eMarketplace can be used to balance the workload between sites with different RIS/PACS, share one global worklist, use dynamic sharing rules (groups of sites, type of data), and generate business reports (who has reported and what). For the customer, it opens up the whole market, providing more choices (but with integrating into only one), allows 24/7 cover through multiple providers, makes it possible to compare prices, availabilities and response times for the reports and to select sub-specialists from a wider pool. For the teleradiology provider, it opens up the whole customer market. In addition, the eMarketplace makes it possible not only to compete by offering lower prices but also to build other competitive advantages like sub-expertise, availability, correctness of the reports, etc. It shows proven quality via the established quality assurance scheme (transparency of the results), makes it easier to integrate the customer and provider systems, and guarantees back-up in the case of a temporary shortage of personnel. The success in implementing many-to-many teleradiology applications also depends on the ability of the service provider not only to solve image reading but also to participate in the planning of procedures to find the best diagnostic approach, to discuss the reports in multidisciplinary meetings and to facilitate clinical communication. Therefore, the teleradiology service has to be implemented in co-operation with the customers.

Fortunately, the EU has taken concrete steps to support the implementation of eHealth and telemedicine, including teleradiology, nationally and across country borders. Interoperable teleradiology and eHealth settings have to follow internationally approved standards and profiles. The standard profiles to solve technical interoperability are XDS and XDS-I. Also, certain certification procedures are being agreed for medical IT systems. 
For the wider use of eHealth, important strategic documents have been recently issued at the European level: the Commission Recommendation on Cross-Border Interoperability of Electronic Health Record System [31], the Commission Communication on Telemedicine for the Benefit of Patients, Healthcare System and Society [1], and the Ministerial Conference Final Declaration about European Co-operation on eHealth [32]. There is also the proposal of the Cross-Border Healthcare Directive, which includes relevant articles about teleradiology.

Those documents include strong recommendations for the implementation of telemedicine and eHealth. For instance, the Member States should have assessed and adapted national regulations to allow telemedicine to be used by the end of 2011. EU authorities underline the importance of the political and strategic commitment of Member States to using eHealth, building confidence and the acceptance of eHealth services, bringing legal and ethical clarity and ensuring protection of personal health data, and solving interoperability issues. It is beneficial for the implementation of new shared teleradiology services when the Commission continues to contribute to European collaboration between health professionals and patients for greater application of telemedicine and makes specific recommendations on how to improve confidence in and acceptance of telemedicine [1].

It is noted that legislation concerning eHealth and teleradiology services in the EU is very complex. The EU takes steps to harmonise national legislation in the area of eHealth. As stated in the treaties of the EU, healthcare follows the principle of subsidiarity [33]. It is the Member State's responsibility to decide on the organisation and delivery of their health services and medical care. Definition of medical acts is a matter for the Member States as well. As a general principle, the classification of specific telemedicine services as medical acts should ensure that these meet the same level of requirements as equivalent non-telemedicine services (e.g. teleradiology vs radiology). This principle ensures that adequately regulated health services are not replaced by less well-regulated telemedicine services and it avoids discrimination between providers of the same service, which would be incompatible with the Directive on Electronic Commerce $[1,20]$.

Concerning the legal regulations of teleradiology and eHealth, the national legislations differ between the Member States. There are various legal approaches regarding teleradiology and eHealth in general: remote archiving of medical data, patients' informed consent, patient claims, etc. Below, there are some examples of differences in legal regulations. Centralised remote archiving is illegal in the Czech Republic, but is an aim in the deployment of the national image archiving solution in France. There are various practicalities in the patients' informed consent to sharing medical data. In some Member States, the physical presence of the patient and the health professional is required, which is a clear obstacle to using teleradiology. In some countries, for example in Austria and Germany, this requirement restricts to practise teleradiology. Moreover, limitations in law or administrative practices often make reimbursement of telemedicine services difficult [1].

Regarding patients' rights, one can usually make a claim against the hospital or another local healthcare provider; in the Netherlands a claim can also be made against the teleradiology provider [20]. The situation is more complex if the teleradiology provider resides outside the EU. In this case the EU legislation does not apply and potential conflicts should be foreseen while making the contract. If the radiologist is reporting outside the EU but works for a company registered in the EU, EU legislation should apply. Yet, clarifications are needed and the market seems to be evolving more rapidly than the directives and laws.

The pace of the delivery of necessary standards and legal certainty must follow a relatively quick time scale because otherwise the rapid development of the eHealth service market would amplify the uncertainty with regard to legal issues and inefficacious interoperability.

\section{References}

1. European Commission. Communication from the Commission to the European Parliament, the Council, the European Economic and Social Committee and the Committee of the Regions on telemedicine for the benefit of patients, healthcare systems and society. http://eur-lex. europa.eu/LexUriServ/LexUriServ.do?uri=CELEX:52008DC0689. EN:NOT (last accessed 16/10/10)

2. The Royal College of Radiologists (2010) Standards for the provision of teleradiology within the United Kingdom. London: The Royal College of Radiologists. http://www.rcr.ac.uk/docs/ radiology/pdf/BFCR\%2810\%297_Stand_telerad.pdf (last accessed $16 / 10 / 10)$

3. Pohjonen H (2010) Images can now cross borders, but what about the legislation? Diagn Imaging Eur 26:4

4. http://www.epsos.eu/ (last accessed 16/10/10)

5. Jarvis L, Stanberry B (2005) Teleradiology: threat or opportunity? Clin Radiol 60:840-845

6. Ross P, Sepper R, Pohjonen H. Cross-border teleradiology experience from two international teleradiology projects. Eur $\mathrm{J}$ Radiol 73:20-25

7. http://www.ihe.net/Profiles/index.cfm (last accessed 16/10/10)

8. McCall I, European Society of Radiology (2010) The future role of radiology in healthcare, European Society of Radiology, 2009, Vienna, Austria. Insights Imaging 1:2-11

9. Benjamin M, Aradi Y, Shreiber R (2010) From shared data to sharing workflow: merging PACS and teleradiology. Eur J Radiol 73:3-9

10. Reponen J (2008) Teleradiology: a Northern Finland perspective. Teleradiology 217-225

11. Størkson S, Aslaksen A (2009) Six months of experience with a XDS-based communication platform for radiology in the Western Norway health care region. Int J Cars 4(Supp 1):168-170

12. http://www.r-bay.org/ (last accessed 16/10/10)

13. http://www.telerays.com/ (last accessed 16/10/10) 
14. http://www.virtualrad.com/ (last accessed 16/10/10)

15. https://www.realtimeradiology.com/ (last accessed 16/10/10)

16. http://www.nighthawkrad.net/ (last accessed 16/10/10)

17. Bradley W (2008) Off-site teleradiology: the pros. Radiology 248:337-341

18. European Society of Radiology. ESR brochure-VII. Teleradiology in the European Union-white paper; November 2006. http://www. myesr.org/cms/website.php?id=/en/publications_media/brochures_i-viii.htm

19. ESR-POC/UEMS Meeting with delegates. Minutes. September 30, 2009 http://www.uemsradiology.eu/meetings-.aspx (last accessed $16 / 10 / 10)$

20. Pattynama P (2010) Legal aspects of cross-border teleradiology. Eur J Radiol 73:26-30

21. Verordnung über den Schutz vor Schäden durch Röntgenstrahlen (Röntgenverordnung). http://www.gesetze-im-internet.de/ r_v_1987/index.html (last accessed 16/10/10)

22. European Commission: Directive 2000/31/EC. http://eur-lex.europa.eu/LexUriServ/LexUriServ.do?uri=CELEX:32000L0031:EN: HTML (last accessed 16/10/10)

23. ECJ Judgements concerning cross border Health Care1998-2006 Summaries. By Roos van der Velde. Based on: "EuGH-Urteile zur grenzüberschreitenden Inanspruchnahme von Gesundheitsdienstleistungen" by Anna Englaender.

24. European Commission: Directive 98/34/EC. http://eur-lex.europa. eu/LexUriServ/LexUriServ.do?uri=OJ:L:1998:204:0037:0048: EN:PDF (last accessed 16/10/10)
25. Proposal for a Directive of the European Parliament and of the Council on the application of patients' rights in cross-border healthcare. http://ec.europa.eu/health/ph_overview/co_operation/ healthcare/docs/COM_en.pdf (last accessed 16/10/10)

26. European Commission: Directive 95/46/EC. http://eur-lex.europa. eu/LexUriServ/LexUriServ.do?uri=CELEX:31995L0046:EN: HTML (last accessed 16/10/10)

27. European Commission: Directive 2002/58/EC. http://eur-lex.europa.eu/ LexUriServ/LexUriServ.do?uri=OJ:L:2006:105:0054:0063:EN:PDF

28. European Commission: Directive 2005/36/EC. http://eur-lex.europa. eu/LexUriServ/LexUriServ.do?uri=OJ:L:2005:255:0022:0142:EN: PDF (last accessed 16/10/10)

29. Healthcare professionals crossing borders, an informal partnership of professional healthcare regulators in Europe. Portugal agreement. Lisbon; 2007. http://admin.uem.net/uploadedfiles/956.pdf (last accessed 16/10/10)

30. Maynard CD (2008) Radiologists: physicians or expert image interpreters? Radiology 248:333-336

31. Commission Recommendation of 2 July 2008 on cross-border interoperability of electronic health record systems. http://eur-lex. europa.eu/LexUriServ/LexUriServ.do?uri=OJ:L:2008:190:0037:0043: EN:PDF (last accessed 05/01/11)

32. Final Conference Declaration. European Co-operation on eHealth. Adopted 15/03/2010. http://www.epractice.eu/en/library/312491 (last accessed 05/01/11)

33. European Commission, Europe Glossary: subsidiarity. http:// www.aer.eu/main-issues/subsidiarity.html (last accessed 16/10/10) 